EPJ Web of Conferences 60, 07008 (2013)

DOI: $10.1051 /$ epjconf/20136007008

(C) Owned by the authors, published by EDP Sciences, 2013

\title{
Searches with long-lived or unusual signatures at LHC
}

\author{
Sigve Haug on behalf of the ATLAS and CMS collaborations ${ }^{1, a}$ \\ ${ }^{1}$ AEC-LHEP University of Bern, Sidlerstrasse 5, CH-3012, Switzerland
}

\begin{abstract}
Several models extending the Standard Model predict final states resulting in long-lived or unusual signatures. Many of these signatures are targeted by the search programs of the Large Hadron Collider experiments ATLAS and CMS. Recent results extracted from the $7 \mathrm{TeV}$ and $8 \mathrm{TeV}$ data, recorded in 2011 and 2012 respectively, are presented. So far no evidence for such signatures have been found and various model exclusions have been performed.
\end{abstract}

\section{Introduction}

Several models extending the Standard Model (SM) predict final states resulting in long-lived or unusual signatures. Long-lived and unusual are here used relatively to SM signatures and refer to signatures for which accelerator operation, data acquisition or detectors are originally not optimized. However, many of these signatures are still addressed by the search programs of the Large Hadron Collider (LHC) experiments ATLAS and CMS [1][2].

At the LHC Physics conference LHCP 2013 recent search results from ATLAS and CMS were presented. They were extracted from the $7 \mathrm{TeV}$ and the $8 \mathrm{TeV}$ data, recorded in 2011 and 2012 respectively. Here, brief signature categorizations are presented, together with their motivations. Then the results from the signature searches are shown. So far no evidence for such signatures have been found and model exclusions have been performed by the experiments.

\section{Signatures and models}

By going from the outer radius of the detectors towards the proton-proton collision point one can categorize the signatures referred to as long-lived or unusual.

- Heavy Stable Charged Particles. In this category the particles are long-lived enough to leave the detector. They differ from muons and neutrinos in the sense that they are heavy compared to their momentum. Thus, they are relatively slow. By measuring the time of flight with the muon spectrometers or the calorimeters this feature can be used as discriminator. Further, if these particles have fractional or multiple charge, they can be selected by their unusual energy loss $d E / d x$ pattern.

- Out-of-time decays. Signatures of particles that stop in the detector, most probably in the calorimeters, and live longer than the typical time between two events at LHC,

\footnotetext{
ae-mail: sigve.haug@lhep.unibe.ch
}

from about 25 to some $100 \mathrm{~ns}$, are referred to as outof-time decays. Such decays can be looked for as jets in later beam crossings, preferably in the empty ones. Due to the beam and run structure of LHC, the typical lifetime sensitivity goes from some $100 \mathrm{~ns}$ to about a day, i.e. to the beam lifetime and the end of a LHC fill.

- Disappearing tracks and displaced vertices. Charged particles that decay during flight in the inner detectors may leave a disappearing track signature. By cleverly exploiting the tracker detectors such signatures can be identified. If no track is reconstructed due to insufficient travel path in the detectors, the decay may be identified as a displaced vertex. Displaced here denotes secondary vertices with larger impact parameters than expected from SM heavy meson decays. As vertex reconstruction algorithms are initially optimized for small impact parameters, adapted reconstruction or special reconstruction efficiency methods have to be used. A certain variant of a displaced vertex is a long-lived neutral particle decaying into a photon. The resulting signature is a photon not pointing towards the collision point. Such events can either be reconstructed by a pointing capability of the electromagnetic calorimeter or by the delayed arrival of the photons.

- Other unusual signatures. A few other signatures not fitting the above categories and being rather prompt were presented at LHCP 2013. They include searches for lepton jets, narrow sprays of first and second generation charged leptons, three jet resonances and heavy resonances decaying into $e \mu, e \tau$ or $\mu \tau$.

Several models extending the SM predict final states resulting in long-lived and unusual signatures as listed above. Hidden-Valley and several supersymmetric (SUSY) models are examples. Moreover, there is a clear experimental and model independent motivation for exploiting the capabilities of the detectors and the recorded data optimally, also by searching for the non-expected. 


\section{Heavy Stable Charged Particles}

A recent and comprehensive search for heavy stable charged particles has been published by CMS in which the full 7 and $8 \mathrm{TeV}$ data sets were exploited [3]. Signal regions were defined by selecting data via muon and missing transverse energy triggers and discriminating variables based on transverse momentum, energy loss and time-offlight measurements from the muon spectrometer. The regions cover fractional and multiple charge, tracker-only, muon-only and tracker plus time-of-flight signatures. The backgrounds were estimated with a data driven method and no significant excess beyond the SM prediction was observed. The interpretations are summarized in Figure 1.

Similar searches were reported by ATLAS with 4.7 $\mathrm{fb}^{-1}$ and $4.4 \mathrm{fb}^{-1}$ of $7 \mathrm{TeV}$ data. In [7], ATLAS uses a signal region defined by $m=p / \gamma \beta$, which is calculated with a time-of-flight and an energy loss measurement, to select heavy sleptons and R-hadrons. In [8], the selection of multiple charged particles is based on the energy loss significance with respect to muons, measured with the monitored drift tubes in the muon system and the transition radiation tracker respectively. The results are indicated in Figure 1.

\section{Out-of-time decays}

CMS has published a search for stopped long-lived particles decaying in subsequent beam crossings with respect to the crossing where the particles would be produced [9]. Such searches are sensitive to lifetimes up to about a day, the upper bound given by the typical lifetime of a LHC proton fill. Empty beam crossings, i.e. without proton bunches, are selected in order to have minimal background and the events are triggered with calorimeter jets. The backgrounds are detector noise, cosmic muons and beam halo events. They are estimated with data driven methods to a total of $8.4 \pm 2.6$ events while the observed number yields 12 events, i.e. a non-significant excess. The full $7 \mathrm{TeV}$ data set was used. In Figure 2 the result is used to show $95 \%$ CL limits on gluino and stop mass as a function of particle lifetime, assuming a cloud model of R-hadron interactions and NLO+NLL production crosssections [10].

ATLAS had to the time of LHCP 2013 not yet published its $8 \mathrm{TeV}$ out-of-time decay analysis. A very similar search can be found in [11].

\section{Disappearing tracks and displaced vertices}

ATLAS has published a search for direct chargino production in anomaly-mediated SUSY breaking (AMSB) models based on a disappearing track signature and the full $7 \mathrm{TeV}$ data set. A motivation for such a search could be $p p \rightarrow \tilde{\chi}_{1}^{ \pm} \tilde{\chi}_{1}^{0} j$. A jet with a high transverse momentum, missing transverse energy and a disappearing track identified by the Transition Radiation Tracker are selected. A background-only fit to the observed transverse momentum distribution yields a $p_{0}=0.54$. The distributions are

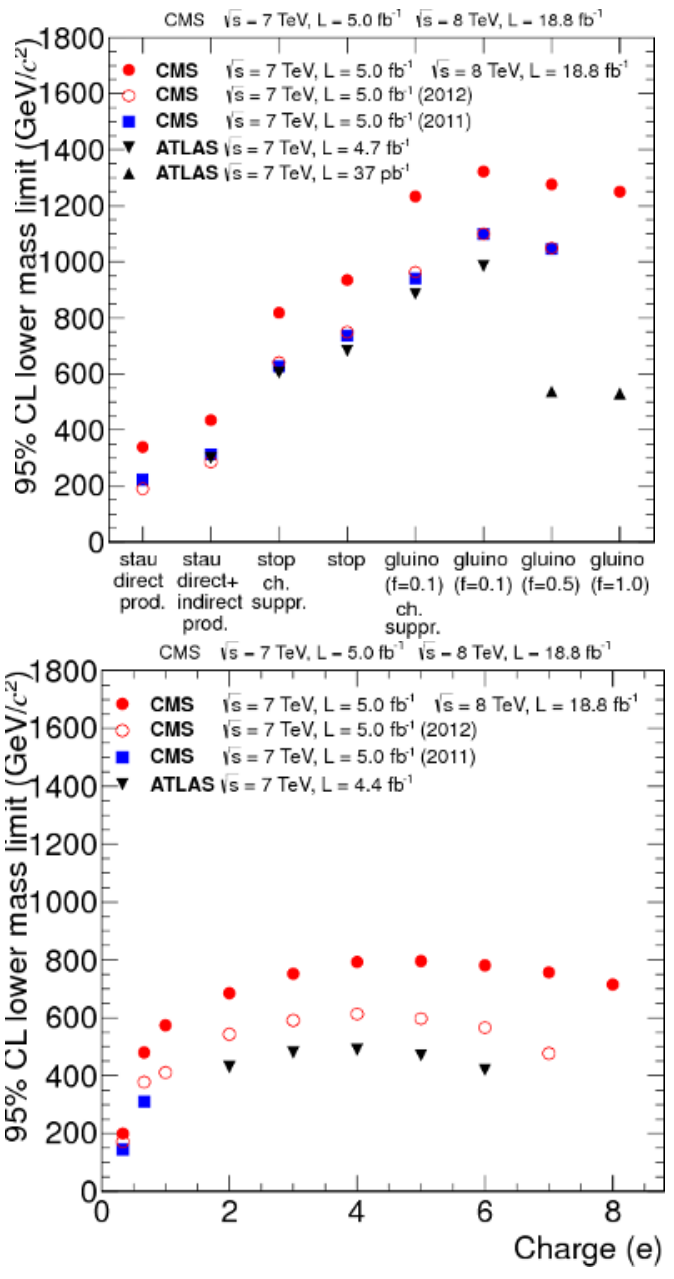

Figure 1. Above, obtained lower mass limits at $95 \% \mathrm{CL}$ on supersymmetric models compared with previously published results. The model type is given on the x-axis. Below, obtained lower mass limits at $95 \%$ C.L. on Drell-Yan like production of fractionally, singly, and multiply charged particles. These particles are assumed to be neutral under $\mathrm{SU}(3)_{C}$ and $\mathrm{SU}(2)_{L}$ [3][4][5][6].

shown in Figure 3. Limits are set on the chargino masses in AMSB models.

Results from displaced vertex searches in the $7 \mathrm{TeV}$ data sets have been published by both ATLAS and CMS [13][14]. They are motivated by a neutralino decaying into a muon and a smuon which again decays in a R-Parity violating mode, and by heavy resonances decaying into longlived neutral particles. Impact parameters of the displaced vertices are used to select signal-like events. Neither ATLAS nor CMS sees any significant deviation from the SM expectation and the results are used to extract mass limits on hypothetical particles.

Both ATLAS and CMS have published results from searches for non-pointing photons, based on the full 7 $\mathrm{TeV}$ data sets [15][16] . For example, in gauge mediated SUSY breaking (GMSB) models the lightest neutralino may decay into a gravitino and a photon. This photon often does not come from the primary interaction region. ATLAS uses the pointing capability of its electromagnetic 


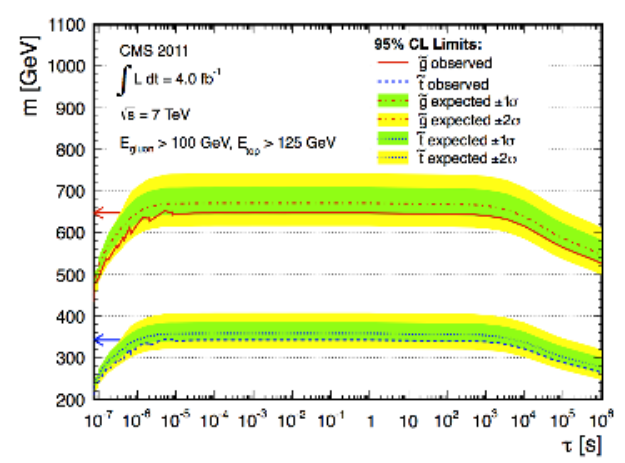

Figure 2. CMS 95\% CL limits on gluino and stop mass as a function of particle lifetime, assuming a cloud model of R-hadron interactions and NLO+NLL production cross-sections [10]. The structure observed between $10^{-7}$ and $10^{-5} \mathrm{~s}$ is due to the number of observed events incrementing when crossing boundaries between lifetime bins [9].

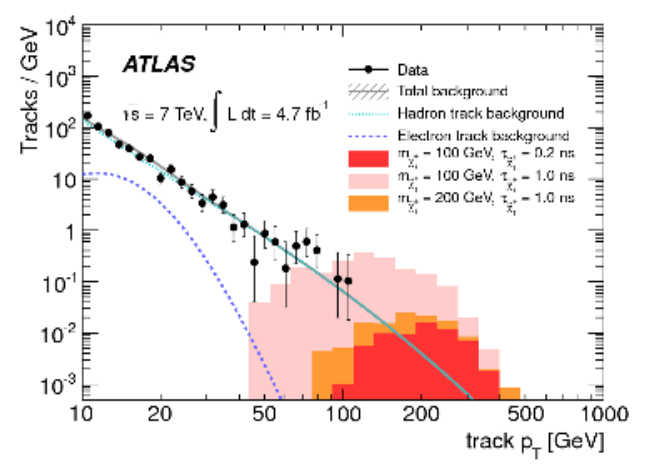

Figure 3. The $p_{T}$ distribution of candidate tracks in the ATLAS disappearing track search [12]. The solid circles show data and lines show background shapes obtained using the backgroundonly fit. The contributions of two background components and the signal expectations are also shown.

calorimeter while CMS relies on the delayed arrival of the photons. The results are consistent with the SM expectations and used to derive mass limits on the neutralino in GMSB.

\section{Other unusual signatures}

ATLAS has performed two searches for lepton-jets in the $7 \mathrm{TeV}$ data set [17][18]. One looked for WH production with a light Higgs boson decaying to a prompt electron-jet, the jet character coming from the boost of the light boson. $\mathrm{A} \mathrm{W}$ is identified together with an electron jet, based on electromagnetic calorimeter properties of the jets. Consistency with the SM expectation was found. In the other search a more inclusive method, simply selecting muon or electron jets, was applied. Also here SM consistency was observed. Several visible cross section limits were published.

At LHCP 2013 an ATLAS search for heavy resonances decaying to $e \mu, e \tau$ or $\mu \tau$ was presented [19]. The search

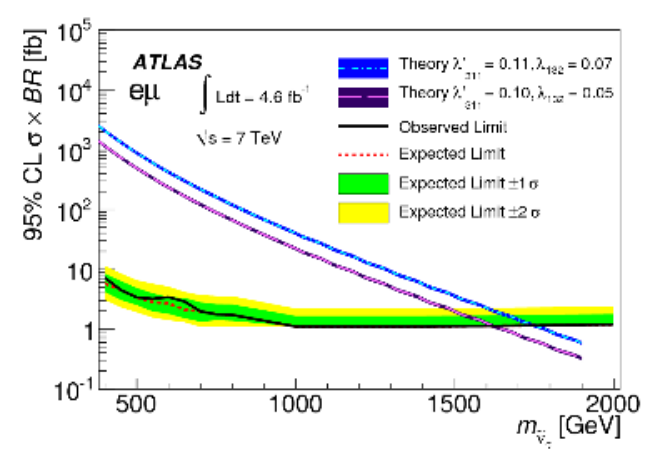

Figure 4. ATLAS 95\% CL upper limit on the production cross section times branching ratio as a function of sneutrino mass for the $e \mu$ mode [19]. The red dotted curve is the expected limit, the black solid curve is the observed limit, and the yellow and green bands give \pm 1 and \pm 2 standard deviations in the expected limit. The expected theoretical curves for $\lambda_{311}^{\prime}=0.11, \lambda_{i 3 k}=$ 0.07 (light blue dot-dash) and $\lambda_{311}^{\prime}=0.10, \lambda_{i 3 k}=0.05$ (light magenta dashed) are also plotted with their uncertainties.

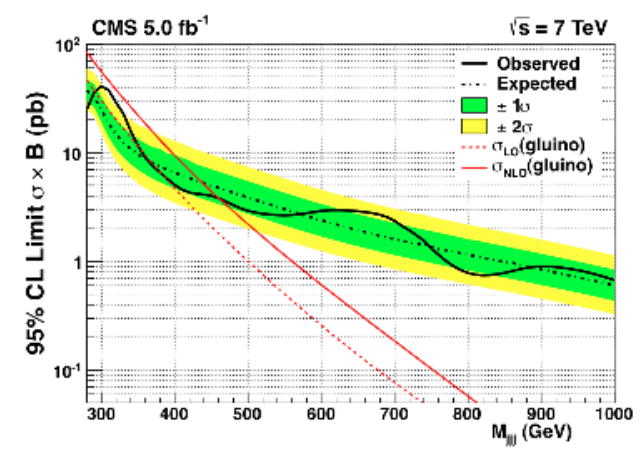

Figure 5. Observed and expected CMS 95\% CL upper limits on the cross section times branching fraction for gluino pair production followed by RPV decay of each gluino to three light-flavored quark jets [20]. Also shown are the $\pm 1 \sigma$ and $\pm 2 \sigma$ bands on the expected limit, as well as the theoretical LO and NLO cross sections for gluino production, assuming a branching fraction of a gluino decay into three jets of $100 \%$.

is motivated by a third generation sneutrino decaying in a lepton flavor violating mode into a pair of leptons with different flavors. The results are based on the $7 \mathrm{TeV}$ data set and in agreement with the SM predications. Mass limits on the $\tilde{v}_{\tau}$ were derived for different R-parity violating (RPV) couplings. An example is shown in Figure 4.

Finally a CMS search for three-jet resonances in the 7 $\mathrm{TeV}$ data was shown [20]. For example, a $\tilde{g} \rightarrow q \tilde{q} \rightarrow q q q$ is a RPV decay sufficiently suppressed if $m_{\tilde{q}}>>m_{\tilde{g}}$. Background was discriminated using the jet-triplet mass. The SM consistent result was used to exclude gluino masses in the benchmark model below $460 \mathrm{GeV}$. The $95 \%$ CL exclusion limit on the visible cross section as a function of the triplet mass is seen to the right in Figure 5. 
with the ATLAS detector, Phys. Lett. B719 (2013) 280298, arXiv:1210.7451 [hep-ex].

[14] CMS Collaboration, Search in leptonic channels for heavy resonances decaying to long-lived neutral particles, JHEP 02 (2013) 085, arXiv:1211.2472 [hep-ex].

[15] ATLAS Collaboration, Search for non-pointing photons in the diphoton and $E_{T}^{\text {miss }}$ final state in $\sqrt{s}=7$ TeV proton-proton collisions using the ATLAS detector, submitted to: Phys. Rev. D, arXiv:1304.6310 [hepex].

[16] CMS Collaboration, Search for long-lived particles in events with photons and missing energy in protonproton collisions at $\sqrt{s}=7 \mathrm{TeV}$, Phys. Lett. 722 (2013) 273, arXiv:1212.1838 [hep-ex].

[17] ATLAS Collaboration, Search for WH production with a light Higgs boson decaying to prompt electron- jets in proton-proton collisions at $\sqrt{s}=7 \mathrm{TeV}$ with the ATLAS detector, New J. Phys. 15 (2013) 043009, arXiv:1302.4403 [hep-ex].

[18] ATLAS Collaboration, A search for prompt leptonjets in pp collisions at $\sqrt{s}=7 \mathrm{TeV}$ with the ATLAS detector, Phys. Lett. B 719 (2013) 299-317, arXiv:1212.5409 [hep-ex].

[19] ATLAS Collaboration, Search for a heavy narrow resonance decaying to $e \mu, e \tau$ or $\mu \tau$ with the ATLAS detector in $\sqrt{s}=7 \mathrm{TeV}$ pp collisions at the LHC, Phys. Lett. B723 (2013) 15-32, arXiv:1212.1272 [hep-ex].

[20] CMS Collaboration, Search for three-jet resonances in pp collisions at $\sqrt{s}=7 \mathrm{TeV}$, Phys. Lett. B 718 (2012) 329, arXiv:1208.2931 [hep-ex]. 